\title{
Feature Recognition of Crop Growth Information in Precision Farming
}

\author{
Hanqing Sun, ${ }^{1}$ Xiaohui Zhang $\mathbb{D}^{2},{ }^{2}$ Zhou Yu, ${ }^{3}$ and Gang Xi ${ }^{4}$ \\ ${ }^{1}$ Department of Electronic and Information Engineering, Henan University of Animal Husbandry and Economy, \\ Zhengzhou 450044, China \\ ${ }^{2}$ College of Electrical Engineering, Henan University of Technology, Zhengzhou 450001, China \\ ${ }^{3}$ School of Information Engineer, Henan Institute of Science and Technology, Xinxiang 453003, China \\ ${ }^{4}$ Department of Applied Physics, Xian University of Technology, Xian 710048, China
}

Correspondence should be addressed to Xiaohui Zhang; xiaohui.xh@163.com

Received 20 June 2018; Accepted 30 September 2018; Published 15 October 2018

Academic Editor: Rongqing Zhang

Copyright (C) 2018 Hanqing Sun et al. This is an open access article distributed under the Creative Commons Attribution License, which permits unrestricted use, distribution, and reproduction in any medium, provided the original work is properly cited.

To identify plant electrical signals effectively, a new feature extraction method based on multiwavelet entropy and principal component analysis is proposed. The wavelet energy entropy, wavelet singular entropy, and the wavelet variance entropy of plants' electrical signals are extracted by a wavelet transformation to construct the combined features. Principal component analysis (PCA) is applied to treat the constructed features and eliminate redundant information among those features and extract features which can reflect signal type. Finally, the classification method of BP neural network is used to classify the obtained feature vectors. The experimental results show that this method can acquire comparatively high recognition rate, which proposed a new efficient solution for the identification of plant electrical signals.

\section{Introduction}

The plant electrical signal is a kind of physiological signal relating to plants' physiological process and their internal information transmission which is a kind of stress response of plants to environmental changes. Electrical signals can not only transmit remote information from point to point on a plant rapidly, but also respond to external stimuli such as light, temperature, burn, and salt sensitively; even these changes take place much earlier than other kinds of physiological and morphological changes usually $[1,2]$. Through the research of plant electrical signals, the information expressed by plants in the natural world is deciphered by human beings. Therefore, an automatic adoptive control system conducted by plant electrical signals is established, which realizes the automatic production controlling system based on the concept of energy-saving and emission-reducing when manufacturing crops and vegetables [3].

With hundreds of years of studying plants' electrical signals, researchers have developed a set of plant signal models and physiological effects relating to this, from the stage where they can only identify the existence of those signals [4]. However, relevant research of this field has not been deeply engaged by domestic and foreign scholars yet. Basic features of plants' electrical signals and their spectral distribution still remain uncertain. Recently, wavelet transformation is widely applied in the research of faint electrical signals such as EEG, ECG, and EMG, but it was seldom introduced to study plants' electrical signals.

As the good time-frequency localization characteristic wavelet transformation is acquired, it is very suitable for nonstationary and time-varying signal analysis; all varieties of wavelet entropy based on this feature are the combination of wavelet transform and the information entropy principle [5]. Wavelet transformation combines the unique advantages in processing irregular signal and the statistical characteristics of figuring the complexity of information entropy. So the wavelet entropy has unique advantages in nonstationary and time-varying signal feature extraction. Although feature information of signal will be more adequate as the feature index increases, the complexity and difficulty of analysis may also be enhanced. In fact, there is a certain information 


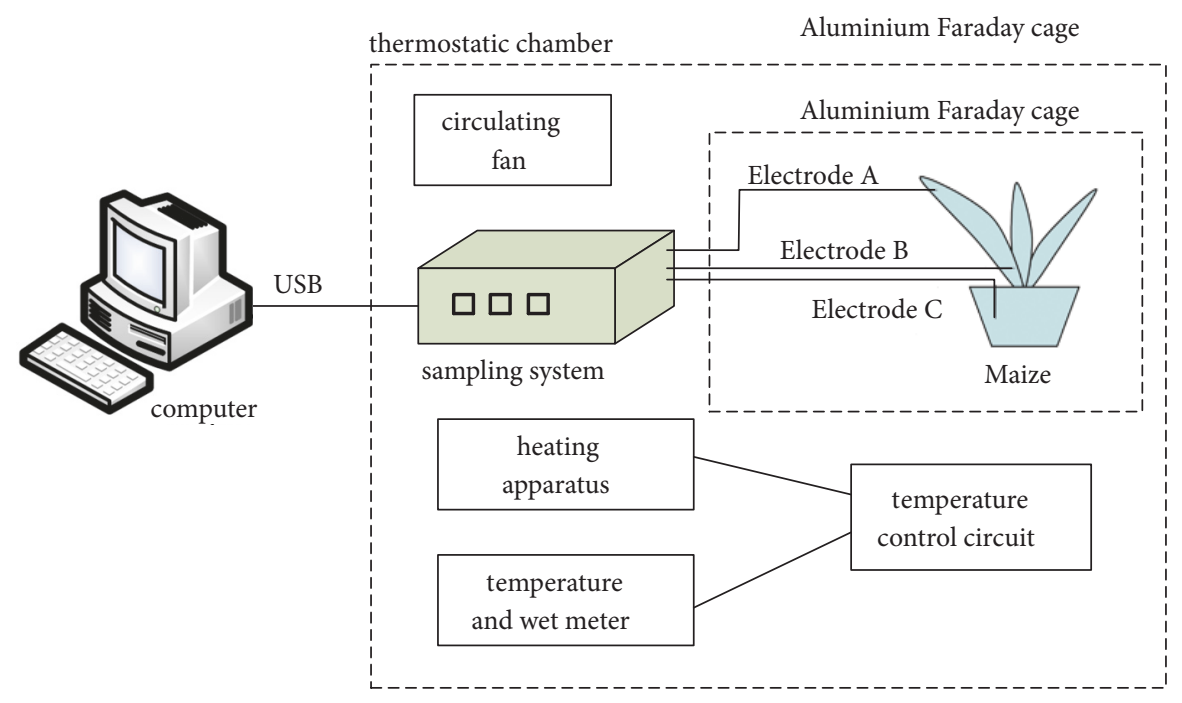

FIGURE 1: Schematic diagram of experimental device.

correlation between different characteristic indexes of a same signal. In order to solve the related problems owing to the increase of information indexes, less independent index will be applied to reflect the information of the original index. We can use the principal component analysis (PCA) as the treatment. The principal component analysis method is used to reduce the number of the original number of evaluation indicators into a small number of comprehensive indicators (principal component), using the idea of dimension reduction. The indexes that are not related to each other can retain the majority of information in the original index, simplifying the complexity of the problem and achieving good results in the process of solving many practical problems. Therefore, the identification of plant electrical signals is proposed, based on the advantages of these two methods.

In this article, the concept of signal wavelet is decomposed and wavelet coefficients are reconstructed first, while transforming the original signal to a number of subsignals that have equal length and contain different frequency; then we extract wavelet energy entropy, wavelet singular entropy, and wavelet variance entropy of each subsignal to construct as the plant electrical feature; then the dimension reduction idea of principal component analysis (PCA) is used to treat a plenty of original data with dimension reduction, eliminating redundant information among features and obtaining a small number of comprehensive principal component indexes independent of each other; finally, identification of plant signal is realized by adopting the method of BP neural network.

\section{Materials and Methods}

2.1. Material and Osmotic Stress. Maize seeds (Zhengdan 985) were provided by the Agricultural Seed Market of Henan. The seeds were germinated on wet filter paper in an incubator where a constant temperature of $30^{\circ} \mathrm{C}$ can be maintained. Once the seeds have germinated, they would be transplanted into a pot filled with soil. The soil contained some essential nutrients to supply the growth of plants; it was composed of $50 \%$ peat, $35 \%$ clay, and $15 \%$ humus $(\mathrm{pH}$ 6.0) and grown under a light intensity of $60 \mu \mathrm{mol} \mathrm{m} \mathrm{m}^{-2} \mathrm{~s}^{-1}$ provided by mercury halide lamps in a $14 / 10 \mathrm{~h}$ light/dark period, at a watering cycle of 3 days with $1 \% \mathrm{KH}_{2} \mathrm{PO}_{4}$ solution. To apply osmotic stress, 15\% PEG6000 was introduced to solve this problem when the maize plants were at the 4-leaf stage. The electrical signals of maize leaves were measured after various periods of sampling under osmotic stress. Corn which grew in a normal condition is set as a comparing group. The leaf relative water content will be measured, and the corresponding electrical signals will be collected when a mild stress, moderate stress, and severe stress are imposed, respectively. About 10 groups of data will be collected in each case.

2.2. Measuring Instrument. A new biological function experiment system (designed by Henan University of Technology, the integrated circuit Laboratory [6]) is introduced to measure electrical signals. The input impedance is $400 \mathrm{M} \Omega$, and every input channel is photo-electrically isolated to decrease outside interference. The equivalent input noise voltage of the instrument was less than $1 \mu \mathrm{V}$. The measuring system is shown in Figure 1. The constant temperature control system, shielding box, and measuring instrument were connected to each other. The sampling frequency of the system and the frequency of the low-pass filter were $5 \mathrm{kHz}$ and $400 \mathrm{~Hz}$, respectively, and the frequency of the high-pass filter was $0.053 \mathrm{~Hz}$. The unit of electrical potential energy is $1 \mu \mathrm{V}$ and the unit of time is seconds. During the sampling, the temperature of the environment was $20^{\circ} \mathrm{C}$ while the humidity was $40-50 \%$.

The electrode was a high-sensitivity silver AMBU ECG electrode. Before the experiment, the results of a test about the electrodes showed that the system can detect signals between 0 and $50 \mathrm{~Hz}$, while the fluctuations were less than $0.5 \%$, which demonstrated the high electrode stability that this system acquires. To measure the plant electrical signal, the A electrode was placed on the front surface of the leaf 
and the B electrode on the back (leaf length, $25 \mathrm{~cm}$; leaf width, $2 \mathrm{~cm}$ ). The distance between two electrodes was 15 $\mathrm{cm}$. The $\mathrm{C}$ electrode was connected to the copper sheet, which was covered by the soil. The measuring electrode was connected to the signal measurement system, and the electrical signal was monitored and recorded by a computer. The environment temperature was regulated by the constant temperature controller [7].

Signal recognition is the differential of the related information of signals, such as the fact that test corn leaves electrical signal were collected at different drought stress. The identified signals are often the same kind of signals. The same kind of signals acquires different information due to different environments or man-made factors; the information of signal only represents a small percentage of all information, but it is the difference between categories of main information signal characteristics. With this idea, the paper is determined to find a suitable method to extract the characteristic of the category information, and we mark the reflected characteristics of the main information of the signal as similar characteristics, while those subtle but being hard to extract features are marked as category. In this paper, the study of the plant electrical signal feature extraction originates from this concept, and the work is divided into two parts: The first step is to find characteristics of the signal information that are clearly presented. The second step is extracting new features based on the first step. The proportion of each attribute characterization of information is different, and the category information characteristics are obtained.

\section{Feature Extraction of Plant Electrical Signals Based on Multiwavelet Entropy}

In the information theory, entropy is a description that describes the degree of uncertainty in the system. If a signal source is treated as a material system, the randomness and uncertainty the signal source will be greater if the output information expands, and the entropy will also be as great as the disorder. This is the reason why the information entropy is regarded as the measurement of system disorder degree [8]. If the coefficient matrix of wavelet transformation is processed as a probability distribution sequence, the sparsity degree of coefficient matrix will be reflected by entropy value obtained from probability distribution sequence, that is, the order degree of signal probability distribution. Nevertheless, the probability distribution to random distribution of signals will be closer since the entropy value is greater [9].

\subsection{Definition of Three Kinds of Wavelet Entropy}

3.1.1. Wavelet Energy Entropy. Wavelet energy entropy is a statistic analysis that analyzes the energy distribution of analyzed signal on each frequency band, using the scale coefficient of wavelet transformation as a benchmark to divide the signal energy. Finally, a quantitative entropy value reflects the distribution complexity of signal energy.

$E=E_{1}, E_{2}, \ldots, E_{m}$ is supposed as wavelet energy spectrum of signal $x(t)$ on $m$ scales; thus a division of signal energy is formed on $E$ scale domain. According to the feature of orthogonal wavelet, the sum power $E$ in a time window is equal to the sum of every component power $E_{j}$. Suppose $p=E_{j} / E(j=1,2, \ldots, m)$; then $\sum p_{j}=1$. Therefore, define wavelet energy entropy as

$$
W_{e}=-\sum_{j=1}^{m} p_{j} \ln \left(p_{j}\right)
$$

$$
p_{j} \ln \left(p_{j}\right)=0 \text { when } p_{j}=0 .
$$

3.1.2. Wavelet Singular Entropy. The theory of singularity value decomposition can easily extract the fundamental modal feature of analyzed matrix. Wavelet singular entropy adopts the advantages of SVD theory to extract analyzed signal's wavelet transformation coefficient matrix feature, reflecting the time domain distribution feature of analyzed signal.

Suppose that signal's wavelet decomposition in the scale $j(j=1,2, \ldots, m)$ is $D_{j(n)}$; thus the decomposition in $m$ scales can constitute an $m \times n$ matrix $D$. According to SVD theory, for an $m \times n$ matrix $D$, there must exist an $m \times l$ dimensional matrix $U$, an $l \times n$ dimensional matrix $V$, and an $l \times l$ dimensional matrix $\Lambda$, decomposing matrix $D$ as

$$
D_{m \times n}=U_{m \times l} \Lambda_{l \times l} V_{l \times n}^{T}
$$

where diagonal matrix $\Lambda$ 's main diagonal element $\lambda_{i}(i=$ $1,2, \ldots, l)$ is not negative and in descending order, that is, $\lambda_{1} \geq \lambda_{2} \geq \cdots \geq \lambda_{l} \geq 0$. These diagonal elements are singular value of wavelet transformation result $D_{m \times n}$. When the signals have no noise or high signal-to-noise ratio, according to signal's singular decomposition theory, their main diagonal singular value will be only a small number but not zero. The signal wavelet decomposition result matrix's singular value also meets similar rules. The less the signal's frequency component, the less the number of wavelet decomposition result's singular value that is not zero. In order to quantify the feature of signal's frequency component and distribution feature, wavelet singular entropy is defined as

$$
W_{s}=\sum_{i=1}^{k} \Delta p_{i}
$$

where $\Delta p_{i}$ is $j_{\text {th }}$ incremental wavelet singular entropy.

$$
\Delta p_{i}=-\left[\frac{\lambda_{i}}{\sum_{j=1}^{l} \lambda_{j}}\right] \log \left[\frac{\lambda_{i}}{\sum_{j=1}^{l} \lambda_{j}}\right]
$$

Treating wavelet transformation result matrix with singular value decomposition is equivalent to mapping wavelet space associated with each other to linearly independent feature space. Wavelet singular entropy can discriminate signals with different time domain distribution in a quantifiable way. The greater the signal's complexity and uncertainty, the greater the value of quantify wavelet singular entropy. 
3.1.3. Wavelet Variance Entropy. For a variance spectrum $\left\{p_{w}(j)=(1 / N)\left\|W_{j}\right\|^{2}\right\}, j=1,2, \cdots m$, obtained from a given signal's wavelet decomposition, among which $W_{j}$ is wavelet coefficient's variance spectrum under $j$ scale. Wavelet variance entropy is defined as follows:

$$
W_{v}=-\sum_{j=1}^{m} p_{j} \ln \left(p_{j}\right)
$$

where $p_{j}=p_{w}(j) / \sum_{j} p_{w}(j)$ indicates the variance probability under $j$ scale.

\subsection{The Feature Extraction Method of Multiple Wavelet} Entropy. Different wavelet entropy reflects signal's distribution statistic feature from different angles. Due to the existence of uncertainty and incompleteness of feature information, especially when the case of error or loss of information occurs during the procession of signal transmission, not every kind of wavelet entropy can completely reflect signal's time-domain feature. Aiming at different types of signals, different kinds of wavelet entropy algorithms have different advantages and features. If we extract, classify, and identify plant electrical signals with single wavelet entropy, it would be hard to deal with signal's uncertainty and changeability interference, which cannot guarantee rightness and reliability of classification. As is shown below, a given signal constructs multiple wavelet entropy feature vectors; detailed steps are as follows.

Step 1. Deal signal $x(n)$ with two-layer wavelet decomposition. Two high-frequency coefficient cd1, cd2 and second layer low-frequency coefficient ca2 are extracted, respectively.

Step 2. Reconstruct wavelet decomposition coefficient and extract signals among every frequency range. $X_{i}(i=1,2,3)$ is used, respectively, to indicate reconstruction signal of cd1, $\mathrm{cd} 2$, and cd3. Therefore, entire reconstruction signal $X$ in two-layer wavelet decomposition can be described as

$$
X=X_{1}+X_{2}+X_{3}
$$

Step 3. Calculate wavelet energy entropy, wavelet variance entropy, and wavelet singular entropy of each reconstruction signal. $W_{e}(i), W_{s}(i)$, and $W_{v}(i)$ are used, respectively, to indicate wavelet energy entropy, wavelet singular entropy, and wavelet variance entropy, among which $i=1,2,3$.

Step 4. Reconstruction feature vector: all three wavelet entropy values are expressed in scientific notation. Extract their effective numbers to construct multiple wavelet entropy's feature vector $T$, which is shown as follows:

$$
T=\left[W_{e 1}, W_{e 2}, W_{e 3}, W_{s 1}, W_{s 2}, W_{s 3}, W_{v 1}, W_{v 2}, W_{v 3}\right]
$$

In this article, db5 wavelet that has compact support and orthogonality is used to treat corn electrical signal with wavelet decomposition, adopting kinds of discussed wavelet entropy extraction algorithm to construct feature vector. This kind of feature vector combines with different entropy's features, thus describing the original signal's information content in a comprehensive way.

\section{Principal Component Analysis of Multiple Wavelet Entropy Feature}

Principal component analysis is not only a statistic analysis method, but also a data compression and feature information extraction method. It will concentrate information which disperses in a set of variables to a few comprehensive indexes (principal component) independent of each other. Every principal component is linear combination of original variables. Every principal component is in an orthogonal relationship with each other, in order to achieve data dimension reduction and redundant information elimination. Set the original sample data of $10 \times 9$ matrix of the multiwavelet entropy feature of the corn in good growing condition as an example. The theory of using principal component analysis to realize data compression and principal component extraction is shown as follows [10].

Step 1. Data standardization process: suppose that the original sample data matrix of the multiwavelet entropy feature of the corn in normal growing condition is $X=(x(i, j))_{10 \times 9}$, among which $i=1,2, \ldots, 10, j=1,2, \cdots 9$, and $x(i, j)$ is the signal's jth feature index.

The expression of data standardization process is

$$
x *(i, j)=\frac{x(i, j)-\bar{x}_{j}}{s_{j}}
$$

where $\bar{x}_{j}$ is sample mean and $S_{j}$ is sample standard deviation. Their calculation expressions are as follows:

$$
\begin{aligned}
& \bar{x}_{j}=\frac{1}{10} \sum_{i=1}^{10} x_{(i, j)} \\
& S_{j}^{2}=\frac{1}{10-1} \sum_{i=1}^{10}\left(x_{(i, j)}-\bar{x}_{j}\right)^{2}
\end{aligned}
$$

After standard treatment, the normalized matrix $X^{*}$ is obtained.

Step 2. Calculate correlation coefficient matrix. Calculate the correlation coefficient matrix $R=\left(r_{(i, j)}\right)_{9 \times 9}$ of normalized matrix $X^{*}$. The calculation expression of $r_{(i, j)}$ is

$$
r_{(i, j)}=\frac{1}{10-1} \sum_{k=1}^{10} x_{(i, k)}^{*} \times x_{(j, k)}^{*}
$$

Step 3. Calculate the feature value and corresponding feature vector of correlation coefficient matrix. Let $|R-\lambda I|=0$ and 9 nonnegative feature value $\lambda_{i}$ can be calculated. They are the variance of principal component. The feature value is sorted by descending order. The corresponding feature vector is set as $l_{1}, l_{2}, \ldots, l_{9}$; thus the $i_{\text {th }}$ principal component expression of original sample data is

$$
y_{i}=x_{(i, j)} l_{i}=l_{i} x_{(i, 1)}+l_{i} x_{(i, 2)}+\cdots l_{i} x_{(i, 9)}
$$

Step 4. Determine the number of principal components. The principle of selected subordinate component is as follows: 


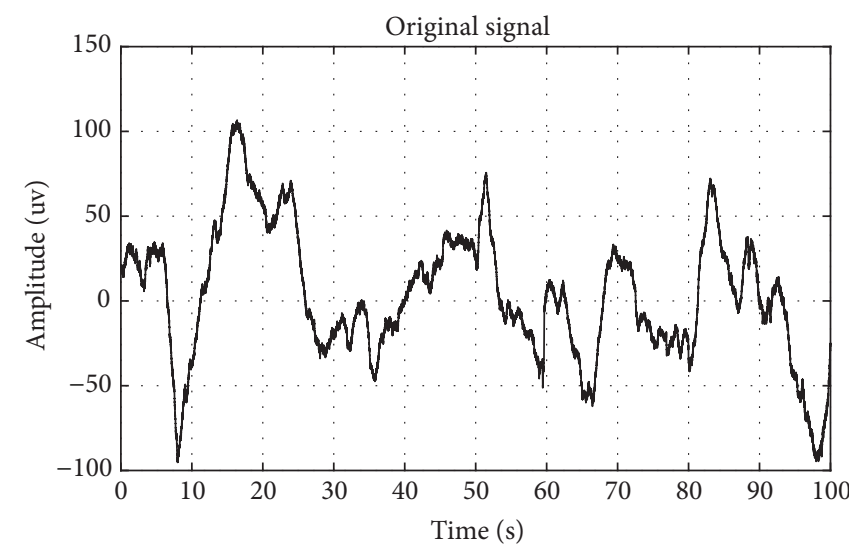

(a)

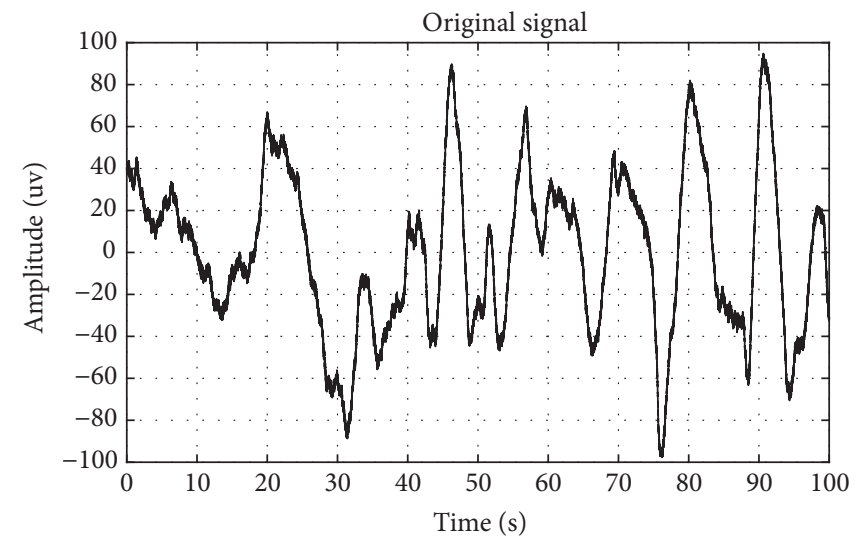

(c)

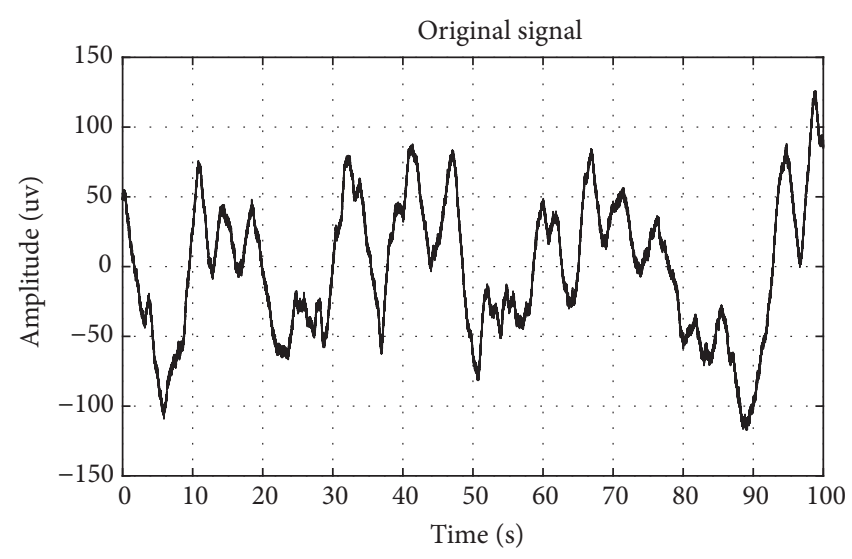

(b)

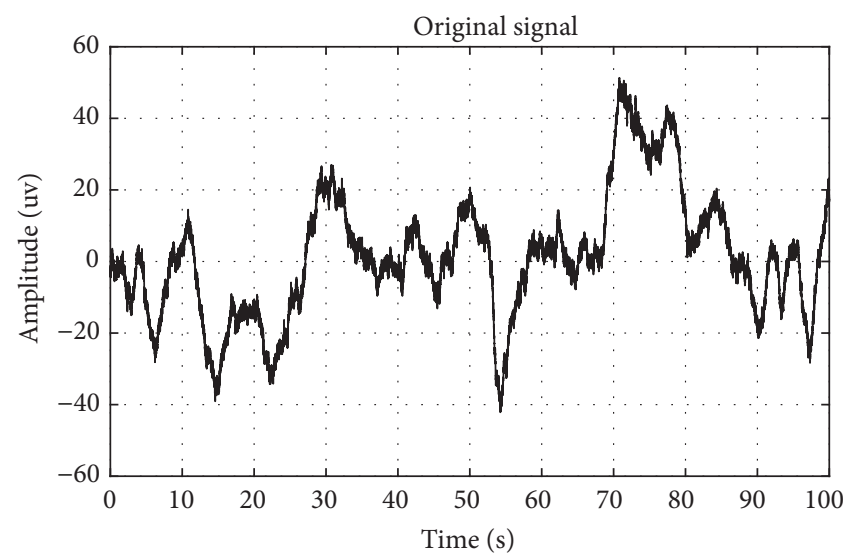

(d)

Figure 2: Time domain waveform of electrical signal of maize leaf under osmotic stress: (a) the normal growing condition; (b) the mild drought stress; (c) the moderate drought stress; (d) the severe drought stress.

original sample date $X$ corresponds to feature value $\lambda_{i}$ and accumulated contribution rate $a=\sum_{i=1}^{p} \lambda i / \sum_{j=1}^{9} \lambda_{j}$ of $p$ principal component is over $85 \%$, indicating this $p$ is the principal component representing the similar feature of original signal. Select the rest 9- $p$ subordinate component as the category feature of identification signal.

\section{Results and Discussion}

5.1. Time Domain Characteristics of Electrical Signal in Maize Leaf under Osmotic Stress. Plant electrical signals are very weak and are easily masked by background noise. Although the measuring instrument and the shield method used in this study can reduce external interference, there is still some background noise. To obtain the most accurate power spectrum of signals in maize leaves, we used a wavelet transform method to denoise the original signal. The time domain waveform of the electrical signal in maize leaf is shown in Figures 2(a), 2(b), 2(c), and 2(d). This waveform was decomposed through a db5 wavelet at seven scales, soft threshold denoised, and reconstructed. The electrical signals were sampled separately at the normal growing condition, the mild drought stress, the moderate drought stress, and the severe drought stress.

5.2. Power Spectrum Analysis of Electrical Signal in Leaf of Maize. Fast Fourier transform was used to analyze the spectrum of the electrical signal in maize leaves. The power spectrum distributions at various intervals under osmotic stress are shown in Figure 3.

5.3. Feature Vector. The original sample date of multiwavelet entropy of corn electrical signal under 4 conditions is $10 \times 9$ matrix, whose feature values are shown in Tables 1, 2, 3, and 4.

As it can be seen from calculation of each table, the accumulated contribution rate of first three principals in normal growing condition group is $85.38 \%$ when $p=3$, the accumulated contribution rate of first three principals in mild drought stress group is $98.02 \%$, the accumulated contribution rate of first three principals in moderate drought stress group is $87.03 \%$, and the accumulated contribution rate of first three principals in severe drought stress group is $96.38 \%$. Therefore, select the last six components as category feature in this 
TABLE 1: The multiwavelet entropy of corn's multiwavelet entropy of the normal growing condition group.

\begin{tabular}{|c|c|}
\hline Number & Feature vector \\
\hline 1 & 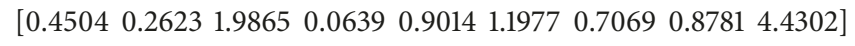 \\
\hline 2 & 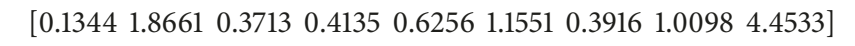 \\
\hline 3 & 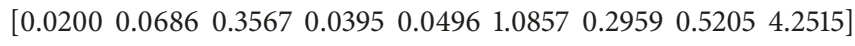 \\
\hline 4 & 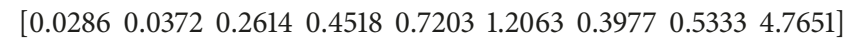 \\
\hline 5 & 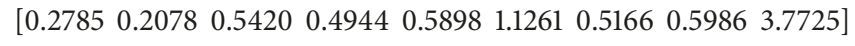 \\
\hline 6 & {$\left[\begin{array}{lllllllll}0.0695 & 0.0492 & 6.9059 & 0.0639 & 0.9014 & 1.1977 & 0.7069 & 0.8781 & 4.4302\end{array}\right]$} \\
\hline 7 & 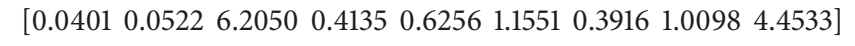 \\
\hline 8 & 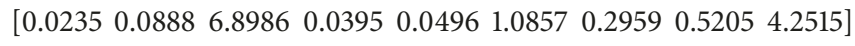 \\
\hline 9 & 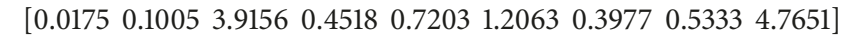 \\
\hline 10 & 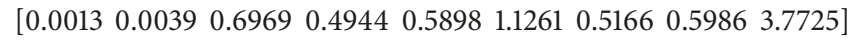 \\
\hline
\end{tabular}

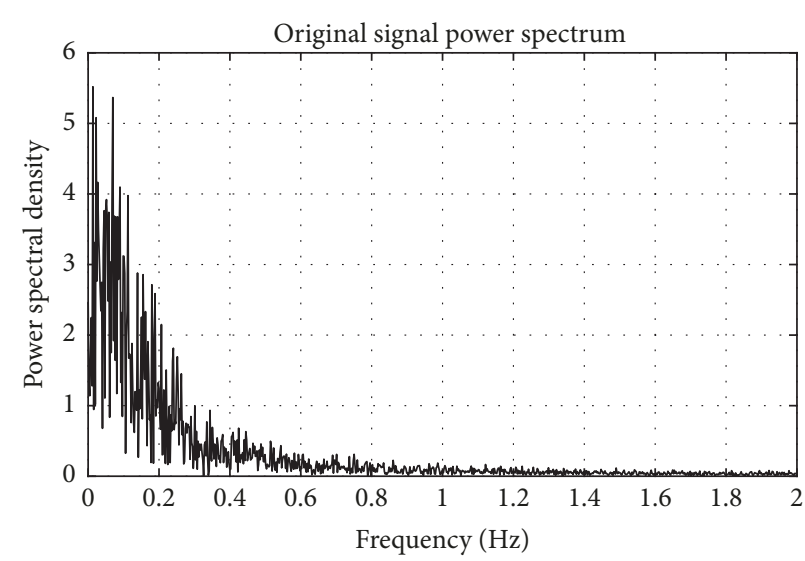

(a)

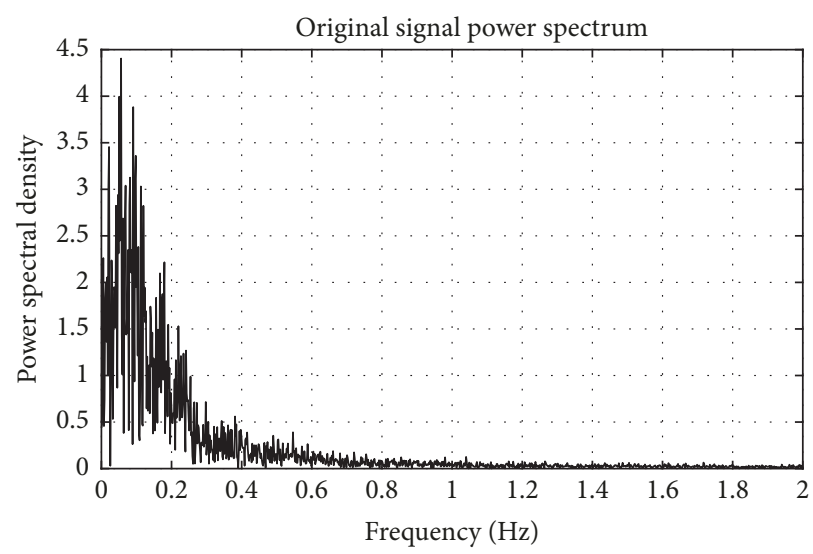

(c)

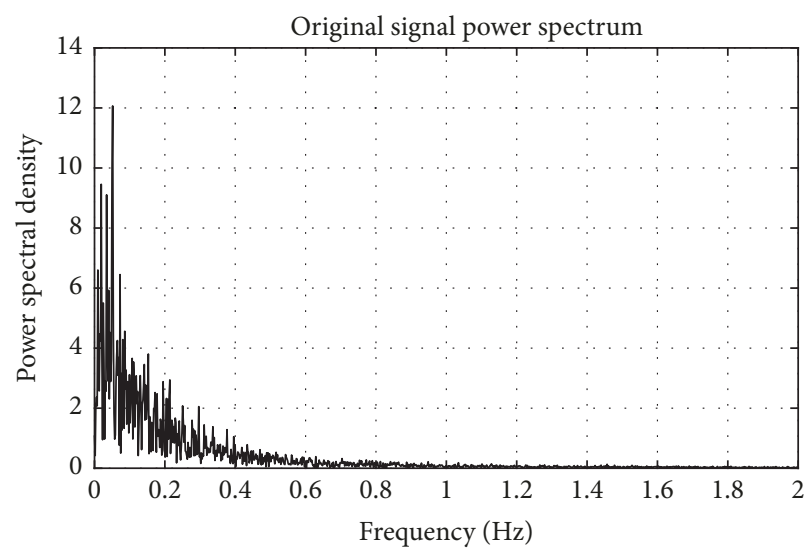

(b)

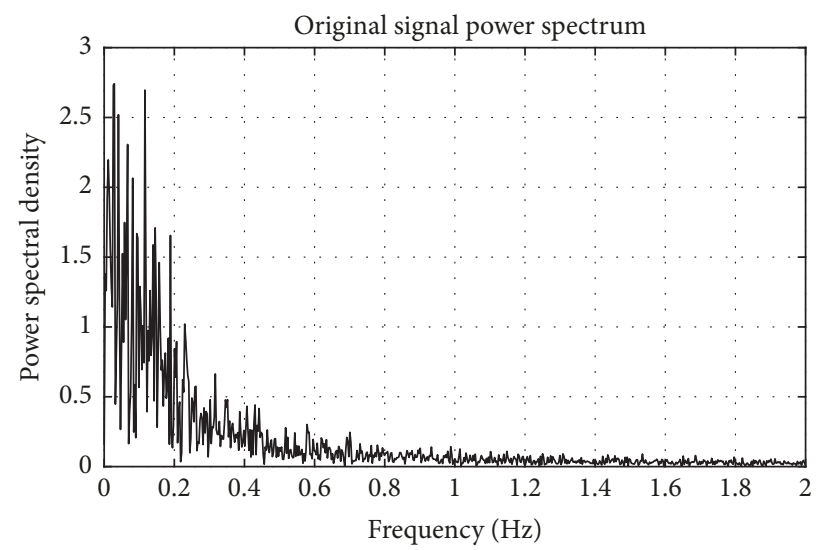

(d)

FIGURE 3: Power spectrum distribution of electrical signals in maize leaf under osmotic stress. (a) The normal growing condition; (b) the mild drought stress; (c) the moderate drought stress; (d) the severe drought stress.

paper and construct feature vector $J=\left[y_{4}, y_{5}, y_{6}, y_{7}, y_{8}, y_{9}\right]$. Not only treat the original multiwavelet entropy feature $T$ with dimension reduction, but also eliminate the redundant information among feature.

Four groups of multiwavelet entropy of corn electrical signal are taken into expression (12) to calculate category feature. The specific calculation process is omitted here. Finally, the category feature can be seen in Tables 5, 6, 7, and 8.
5.4. Classification of Feature Value by BP Neural Network. Feature extraction is that multiwavelet entropy of plant electric signal is constructed as feature vector, through principal component analysis to get a few independent principal component indexes and extract the important information which has classification significance. However, classifier design is aimed at making feature vector fall into different categories and use it realize the automatic identification of signals. 
TABLE 2: The feature vector of corn's multiwavelet entropy of the mild drought stress group.

\begin{tabular}{|c|c|}
\hline Number & Feature vector \\
\hline 1 & 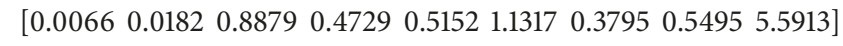 \\
\hline 2 & 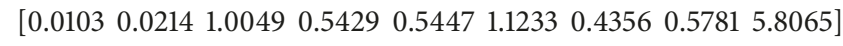 \\
\hline 3 & 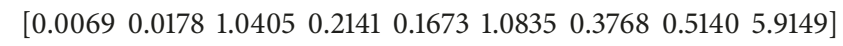 \\
\hline 4 & 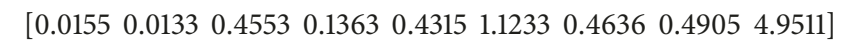 \\
\hline 5 & 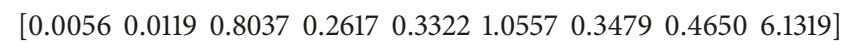 \\
\hline 6 & 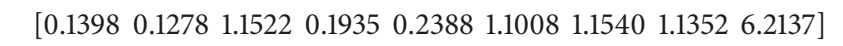 \\
\hline 7 & {$\left[\begin{array}{lllllllll}0.1211 & 0.1288 & 1.1065 & 0.2428 & 0.3004 & 1.1009 & 1.1561 & 1.2566 & 5.6836\end{array}\right]$} \\
\hline 8 & 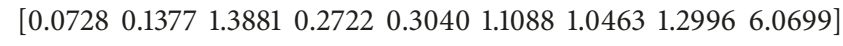 \\
\hline 9 & 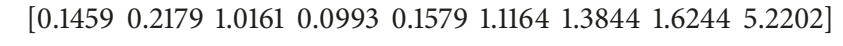 \\
\hline 10 & 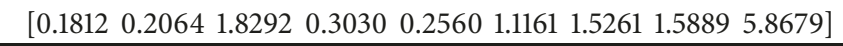 \\
\hline
\end{tabular}

TABLE 3: The feature vector of corn's multiwavelet entropy of the moderate drought stress group.

\begin{tabular}{|c|c|}
\hline Number & Feature vector \\
\hline 1 & {$\left[\begin{array}{lllllllll}0.0323 & 0.0670 & 6.0548 & 0.3542 & 0.3943 & 1.0624 & 0.716 & 0.988 & 8.540\end{array}\right]$} \\
\hline 2 & 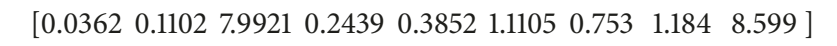 \\
\hline 3 & 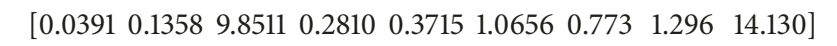 \\
\hline 4 & 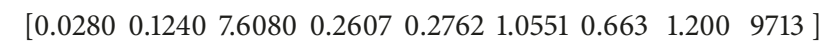 \\
\hline 5 & 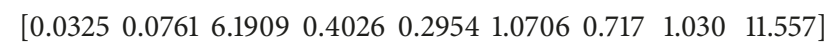 \\
\hline 6 & 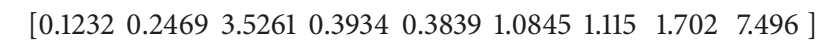 \\
\hline 7 & {$\left[\begin{array}{lllllllll}0.0657 & 0.1520 & 2.8382 & 0.1886 & 0.3792 & 1.1150 & 0.970 & 1.398 & 8.912\end{array}\right]$} \\
\hline 8 & 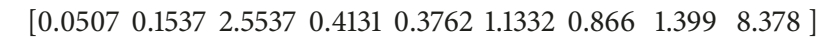 \\
\hline 9 & 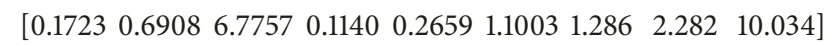 \\
\hline 10 & 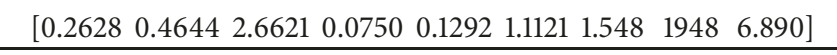 \\
\hline
\end{tabular}

TABLE 4: The feature vector of corn's multiwavelet entropy of the severe drought stress group.

\begin{tabular}{|c|c|}
\hline Number & Feature vector \\
\hline 1 & 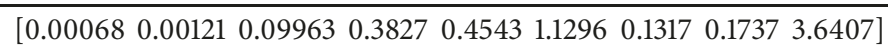 \\
\hline 2 & 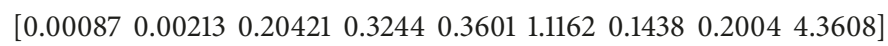 \\
\hline 3 & 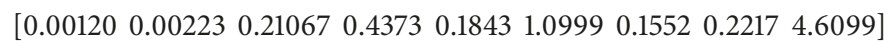 \\
\hline 4 & 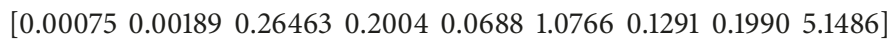 \\
\hline 5 & 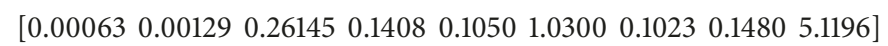 \\
\hline 6 & 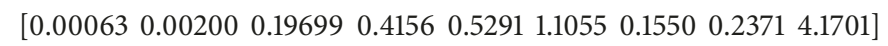 \\
\hline 7 & 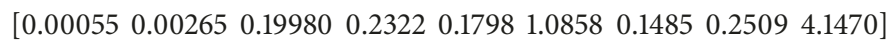 \\
\hline 8 & 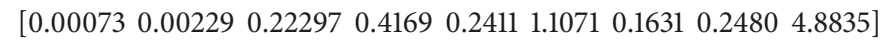 \\
\hline 9 & 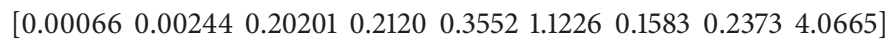 \\
\hline 10 & 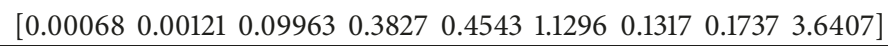 \\
\hline
\end{tabular}

TABLE 5: The category feature of multiwavelet entropy of the normal growing condition group.

\begin{tabular}{|c|c|c|c|c|c|c|}
\hline number & $y_{4}$ & $y_{5}$ & $y_{6}$ & $y_{7}$ & $y_{8}$ & $y_{9}$ \\
\hline 1 & -0.2812 & -0.8892 & 0.0426 & -0.0577 & -0.0938 & -0.4399 \\
\hline 2 & -0.2794 & -0.7743 & -0.0345 & -0.0013 & -0.0746 & -0.4392 \\
\hline 3 & 0.2295 & -0.9332 & -0.1047 & -0.0625 & -0.0756 & -0.4615 \\
\hline 4 & -1.3926 & -0.2681 & 0.0208 & 0.1096 & -0.2003 & -0.4198 \\
\hline 5 & -1.0738 & -0.2396 & 0.2120 & -0.0980 & 0.0868 & -0.4322 \\
\hline 6 & -0.3667 & -0.1592 & -0.4541 & 0.1472 & -0.0194 & -0.4456 \\
\hline 7 & -0.5042 & -0.2346 & -0.0207 & -0.4478 & -0.1281 & -0.4506 \\
\hline 8 & 0.5475 & -0.8620 & 0.0654 & -0.0250 & -0.0579 & -0.4015 \\
\hline 9 & -0.8971 & -0.8595 & 0.2648 & 0.1679 & -0.0774 & -0.4676 \\
\hline 10 & 1.8073 & 1.3733 & 0.2552 & 0.1580 & 0.0392 & -0.4297 \\
\hline
\end{tabular}


TABLE 6: The category feature of multiwavelet entropy of the mild drought stress group.

\begin{tabular}{|c|c|c|c|c|c|c|}
\hline number & $y_{4}$ & $y_{5}$ & $y_{6}$ & $y_{7}$ & $y_{8}$ & $y_{9}$ \\
\hline 1 & -0.2863 & -0.1324 & -0.3372 & -0.0581 & -0.4100 & 0.0560 \\
\hline 2 & 0.0866 & -0.3911 & -0.5060 & -0.1182 & -0.4214 & 0.0496 \\
\hline 3 & -0.2630 & 0.0064 & -0.3462 & -0.0718 & -0.4266 & 0.0518 \\
\hline 4 & -0.4307 & -0.4297 & -0.2906 & -0.1373 & -0.4320 & 0.0521 \\
\hline 5 & -0.4011 & -0.3872 & -0.4298 & -0.0396 & -0.3356 & 0.0587 \\
\hline 6 & 0.0366 & -0.2714 & -0.2837 & -0.0329 & -0.4122 & 0.0680 \\
\hline 7 & -0.0657 & -0.1786 & -0.2772 & -0.0655 & -0.3552 & 0.0413 \\
\hline 8 & -0.2298 & -0.1403 & -0.3610 & -0.2325 & -0.3782 & 0.0601 \\
\hline 9 & -0.3487 & -0.0889 & -0.5159 & -0.0504 & -0.4168 & 0.0549 \\
\hline 10 & -0.3349 & -0.3828 & -0.3315 & -0.0537 & -0.4353 & 0.0507 \\
\hline
\end{tabular}

TABLE 7: The category feature of multiwavelet entropy of the moderate drought stress group.

\begin{tabular}{|c|c|c|c|c|c|c|}
\hline number & $y_{4}$ & $y_{5}$ & $y_{6}$ & $y_{7}$ & $y_{8}$ & $y_{9}$ \\
\hline 1 & 0.9414 & 0.6474 & 0.2574 & -0.4190 & 0.3745 & 0.2405 \\
\hline 2 & 0.3360 & -0.1557 & -0.2745 & -0.2965 & 0.3195 & 0.2357 \\
\hline 3 & 0.6969 & 0.4995 & -0.4853 & -0.4738 & 0.4105 & 0.2498 \\
\hline 4 & 1.0683 & 0.0847 & -0.2928 & -0.3047 & 0.3920 & 0.2459 \\
\hline 5 & 0.6588 & 0.7405 & -0.6613 & -0.4388 & 0.4190 & 0.2314 \\
\hline 6 & -0.5759 & 0.7748 & -0.1728 & -0.1996 & 0.4344 & 0.2428 \\
\hline 7 & -0.5079 & -0.0462 & -0.0469 & -0.5216 & 0.4746 & 0.2400 \\
\hline 8 & -0.5131 & 0.5449 & -0.4015 & -0.4714 & 0.2604 & 0.2438 \\
\hline 9 & 0.3912 & 0.5855 & 0.2801 & -0.4311 & 0.3504 & 0.2390 \\
\hline 10 & 0.5646 & 0.4422 & -0.2028 & -0.3969 & 0.3800 & 0.2410 \\
\hline
\end{tabular}

TABLE 8: The category feature of multiwavelet entropy of the severe drought stress group.

\begin{tabular}{|c|c|c|c|c|c|c|}
\hline number & $y_{4}$ & $y_{5}$ & $y_{6}$ & $y_{7}$ & $y_{8}$ & $y_{9}$ \\
\hline 1 & -0.0270 & 0.4450 & -0.1020 & -0.0116 & 0.0866 & -0.0099 \\
\hline 2 & -0.5460 & 0.6614 & -0.0286 & -0.0113 & 0.0848 & -0.0098 \\
\hline 3 & 0.4023 & 0.4779 & -0.1013 & 0.0053 & 0.1001 & -0.0079 \\
\hline 4 & -0.0346 & 0.3827 & -0.1615 & 0.0096 & 0.0730 & -0.0169 \\
\hline 5 & -0.0130 & 0.2228 & -0.0203 & -0.0410 & 0.0971 & -0.0069 \\
\hline 6 & -0.3268 & 0.1186 & -0.1151 & -0.0051 & 0.0781 & -0.0099 \\
\hline 7 & -0.1094 & 0.5213 & -0.2182 & -0.0330 & 0.0792 & 0.0006 \\
\hline 8 & 0.1391 & 0.5472 & -0.0945 & -0.0455 & 0.0780 & -0.0187 \\
\hline 9 & -0.3049 & 0.4655 & -0.1590 & -0.0022 & 0.1151 & -0.0129 \\
\hline 10 & 0.0512 & 0.4925 & -0.0262 & 0.0150 & 0.0757 & -0.0040 \\
\hline
\end{tabular}

In practical applications, the number of nodes input layers in the network should be determined according to the dimensions of the input data, and the number of nodes output layers in the network is according to the design of the classifier requirement in general. If the network is used as classifier, the number of node output layers should be to classification categories. The number of hidden layers can use one, two, or more hidden layers to construct the network structure. The more hidden layers of network, the stronger the mapping function, but the training time is very long. Number of hidden layer nodes selected is too little, the network will not have enough freedom to better fit the training data, the network is not strong or not training to come out, and then it cannot even identify samples that have not been met before, where fault tolerance is poor. If the number of hidden layer nodes is too high, the network training time is too long, and the error is not necessarily the best. In general, it can only rely on experience and test to determine the number of hidden layer nodes.

BP network is a kind of multilayer network of forward propagation. Apart from having input layer and output layer, it also has one or more hidden layers. A 3-layer BP neural network can be done for any n-dimensional to m-dimensional mapping $[2,11-16]$. The network training 
TABLE 9: The experimental result of BP neural network.

\begin{tabular}{|c|c|c|c|c|c|}
\hline Signal type & normal growing group & mild drought stress group & moderate drought stress group & severe drought stress group & Average value \\
\hline Identification rate & $83.3 \%$ & $100 \%$ & $91.67 \%$ & $100 \%$ & $93.75 \%$ \\
\hline
\end{tabular}

method is called the error backpropagation method. It makes use of the minimum error between the actual output of the network and the expected output (the target vector) to the network's multilayer connection weights and then after the forward layer by layer correction. The method of successive correction of the weight vector is called successive correction method.

Four sets of feature vectors for each class of signals are selected randomly in the experiment, which are taken into BP neural network to process 20000 times learning, and the error is close to 0.01 , and the structure of selection is 3layer BP neural network 4-15-4. The transfer function of the computing unit in the network is trained by the traingdm type function. The results of the experiment are shown in Table 9.

5.5. Analysis of Result. The proposed method is applied to classify the electrical signals of maize leaves in four cases. It could be seen that the recognition rate of the normal growing group and the moderate drought stress group was lower than that in the mild drought stress group and the severe drought stress group. Similarly, it can be seen by the contribution rate in the normal growth and moderate drought stress groups of similar information contained in the class features compared to the other two groups. So the category feature is not obvious, and recognition rate is not as that of the two groups. In general, the average recognition rate of the method is $93.75 \%$, and the recognition rate of it is not only higher than that of just using multiwavelet entropy structure feature; recognition rate is $83 \%$. And the running efficiency of the program is fast, more than half $[17,18]$.

When this method is classified in BP neural network, it will be able to achieve the set target for each training 70008000 times. And when the feature of multiwavelet entropy is identified, training 20000 times to achieve the set target probability is only $90 \%$. It can be seen that it is feasible to use the multiwavelet entropy and principal component analysis to identify the plant electrical signals. Multiwavelet entropy can characterize the information contained in plants' electrical signals. The principal component analysis can not only reduce the dimension of feature vectors and remove redundant information, but also get the classification features of the plant signal, which makes the recognition rate of the classifier greatly improved.

This method also has the advantages of practical application, and the common signal recognition method is the identification of a single signal, but this method is to identify a set of signal samples at the same time. In facility agriculture, it is obviously inappropriate for the detection of a signal to represent a regional crop. Therefore, a large number of repeated experiments are required to calculate the features of the regional crop. A regional crop signal is correlated; as a result, the duplicate information was also tested in repeated trials, and this does not conform to the principle of efficiency. The method proposed in this paper solves this problem. Not only can it identify the data samples of a region at the same time, but also the principal component analysis has removed the correlation between signals. In addition, it has high recognition rate, which can make the early warning and the diagnosis of the plant under the external environment stress more effective.

\section{Conclusion}

In view of the recognition problems of weak, complex, and strong random plant electrical signals, a signal feature extraction method based on multiwavelet entropy and principal component analysis is proposed; the conclusions can be drawn as follows.

(1) All kinds of wavelet entropy are organic combination consisting of wavelet transformation, entropy principle, and related theories; it is a blend of three unique advantages in signal processing; thus it can extract and analyze the nonstationary signals effectively; fusion feature of wavelet entropy can make a good characterization to all of information of plant electrical signal.

(2) Make full use of wavelet transform and principal component analysis. Wavelet transformation time-frequency resolution features are used for signal refinement and signal feature extraction in frequency. Using principal component analysis can not only convert multiwavelet entropy feature index to a handful of independent indexes while eliminating redundant information among features, but also pass through the contribution degree classifying main information and secondary information of the signal. Therefore, we can obtain the class feature, reduce the eigenvector dimensions, simplify the classifier design, and improve the classifier recognition rate.

(3) The proposed approach is used to recognize a variety of conditions of corn leaf electrical signals, it obtains satisfactory results of this classification, and the experimental results verify the feasibility and the validity of this method.

To sum up, more plants for more extensive research shall be used in further study, while the application scope of this method shall be broadened, and we should make more sufficient data mining of plant electric signals to extract the better feature parameters. Based on the above, we have built a practical recognition system of plant electric signals successfully.

\section{Data Availability}

The data used to support the findings of this study are available from the corresponding author upon request. 


\section{Conflicts of Interest}

The authors declare that they have no conflicts of interest.

\section{Acknowledgments}

This study was funded by the National Natural Science Foundation of China (31471412) and supported by the Fundamental Research Funds for the Henan Provincial Colleges and Universities in Henan University of Technology (2016QNJH02) and funded by the Henan Provincial Department of Education Natural Science Project (19B120001).

\section{References}

[1] L. A. Gurovich and P. Hermosilla, "Electric signalling in fruit trees in response to water applications and light-darkness conditions," Journal of Plant Physiology, vol. 166, no. 3, pp. 290300, 2009.

[2] X. Yan, Z. Wang, L. Huang et al., "Research progress on electrical signals in higher plants," Progress in Natural Science, vol. 19, no. 5, pp. 531-541, 2009.

[3] L.-Z. Wang and A.-Y. Hu, "A vista on growth information transmissions of weak electrical signals in plants," China Jiliang University News, vol. 21, no. 3, pp. 192-200, 2010.

[4] L.-z. Wang, D.-S. Li, and L. Qiao, "Research progress of plant electrical signal test," China Jiliang University News, vol. 19, no. 1, pp. 10-18, 2008.

[5] O. Rossoa, A. Blancoa, S. Yordanovab J et al., "Wavelet entropy: a new tool for analysis of short duration brain electrical signals," Journal of Neuroscience Methods, vol. 105, no. 1, pp. 65-75, 2001.

[6] M. Xiao-li, X. Gang, Z. Xiao-hui, and K. Liu, "Analysis of Characteristics of Electrical Signals in the Leaves of Three Species of Plants," Journal of Xian University of Technology, vol. 27, no. 2, pp. 209-213, 2011.

[7] X. H. Zhang, N. M. Yu, G. Xi, and X. L. Meng, "Changes in the power spectrum of electrical signals in maize leaf induced by osmotic stress," Chinese Science Bulletin, vol. 57, no. 4, pp. 413420, 2012.

[8] I. Rezek and S. Roberts, "Stochastic complexity measures for physiological signal analysis," IEEE Transactions on Biomedical Engineering, vol. 45, no. 9, pp. 1186-1191.

[9] H. Hong-hua and Gui Z.-G., "Application of wavelet entropy in denoising processing and R wave detection of ECG signal," Computer Engineering and Applications, vol. 46, no. 9, pp. 116119, 2010.

[10] Z. Ai-hua and Ling-jie K., "Wavelet packet transform and principal component analysis for identifying sub-health state," Computer Engineering and Applications, vol. 47, no. 26, pp. 238241, 2011.

[11] G. Zhe-xue and S. Zhi-qiang, Neural network theory and MATLAB2007 implementation, Electronic Industry Publishing House, Beijing.

[12] A. G. Volkov, C. L. Vilfranc, V. A. Murphy et al., "Electrotonic and action potentials in the Venus flytrap," Journal of Plant Physiology, vol. 170, no. 9, pp. 838-846, 2013.

[13] P. Oyarce and L. Gurovich, "Evidence for the transmission of information through electric potentials in injured avocado trees," Journal of Plant Physiology, vol. 168, no. 2, pp. 103-108, 2011.
[14] L. Wang and Q. Li, "RBF neural networks analysis on electrical signal in chrysanthemum coronarium," in Proceedings of the 2010 6th International Conference on Natural Computation, ICNC'10, pp. 1446-1450, China, August 2010.

[15] D.-J. Zhao, Z.-Y. Wang, J. Li et al., "Recording extracellular signals in plants: A modeling and experimental study," Mathematical and Computer Modelling, vol. 58, no. 3-4, pp. 556-563, 2013.

[16] L. Jingxia and D. Weimin, The feature exraction of plant electrical signal based on wavelet packet and nature network.

[17] E. F. Cabral, P. C. Pecora, A. I. C. Arce, A. R. B. Tech, and E. J. X. Costa, "The oscillatory bioelectrical signal from plants explained by a simulated electrical model and tested using Lempel-Ziv complexity," Computers and Electronics in Agriculture, vol. 76, no. 1, pp. 1-5, 2011.

[18] Y. Qin, L. Huang, A. Liu et al., "Visualization of synchronous propagation of plant electrical signals using an optical recording method," Mathematical and Computer Modelling, vol. 58, no. 34, pp. 661-669, 2013. 


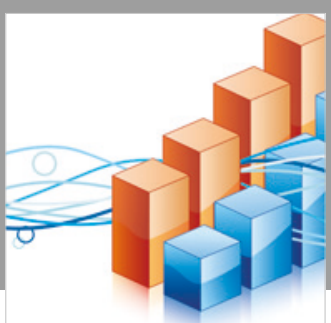

Advances in

Operations Research

\section{-n-m}
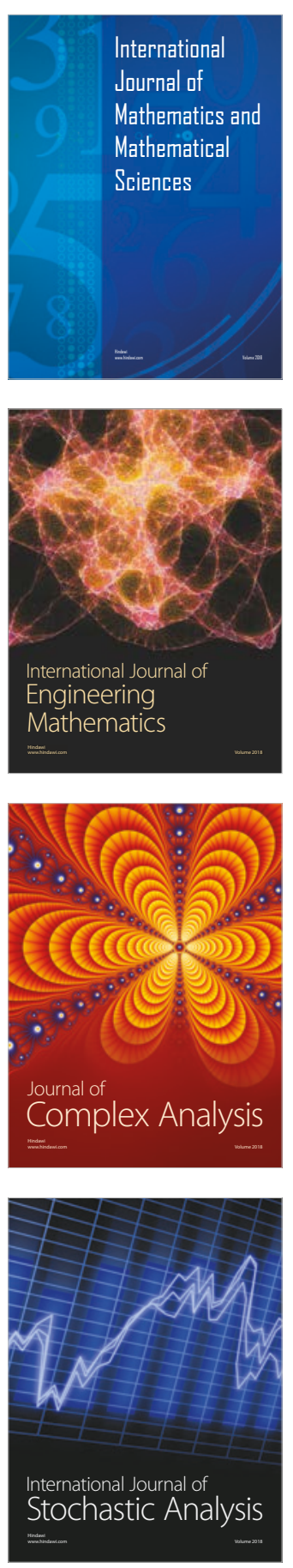
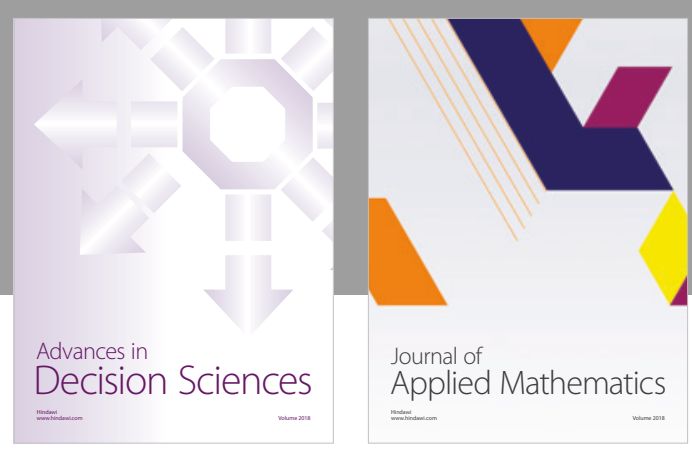

Journal of

Applied Mathematics
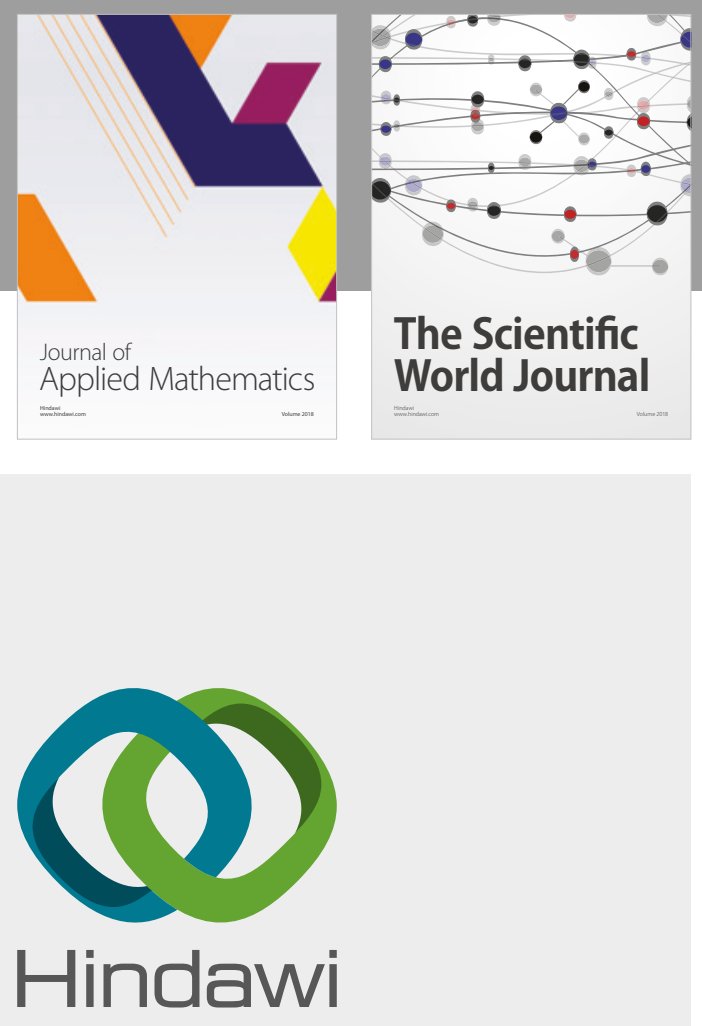

Submit your manuscripts at

www.hindawi.com

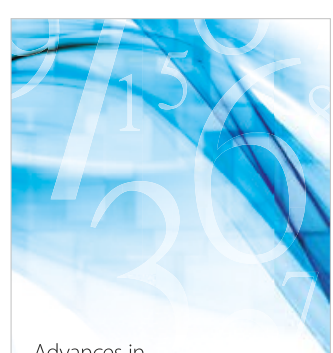

Advances in
Numerical Analysis
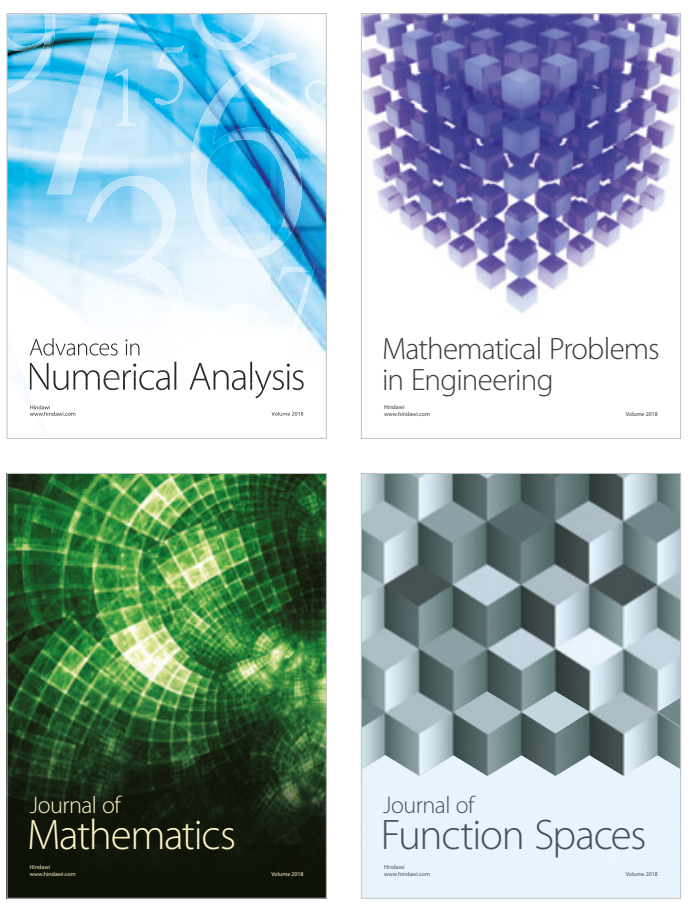

Mathematical Problems in Engineering

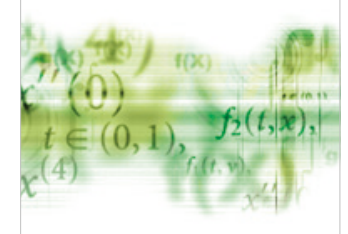

International Journal of

Differential Equations

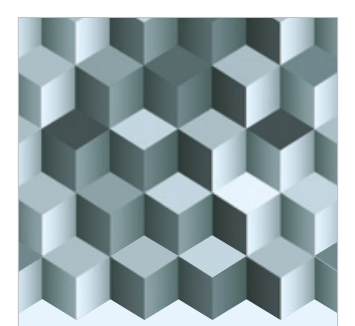

Journal of

Function Spaces
The Scientific

World Journal

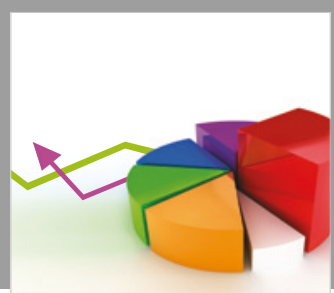

Journal of

Probability and Statistics
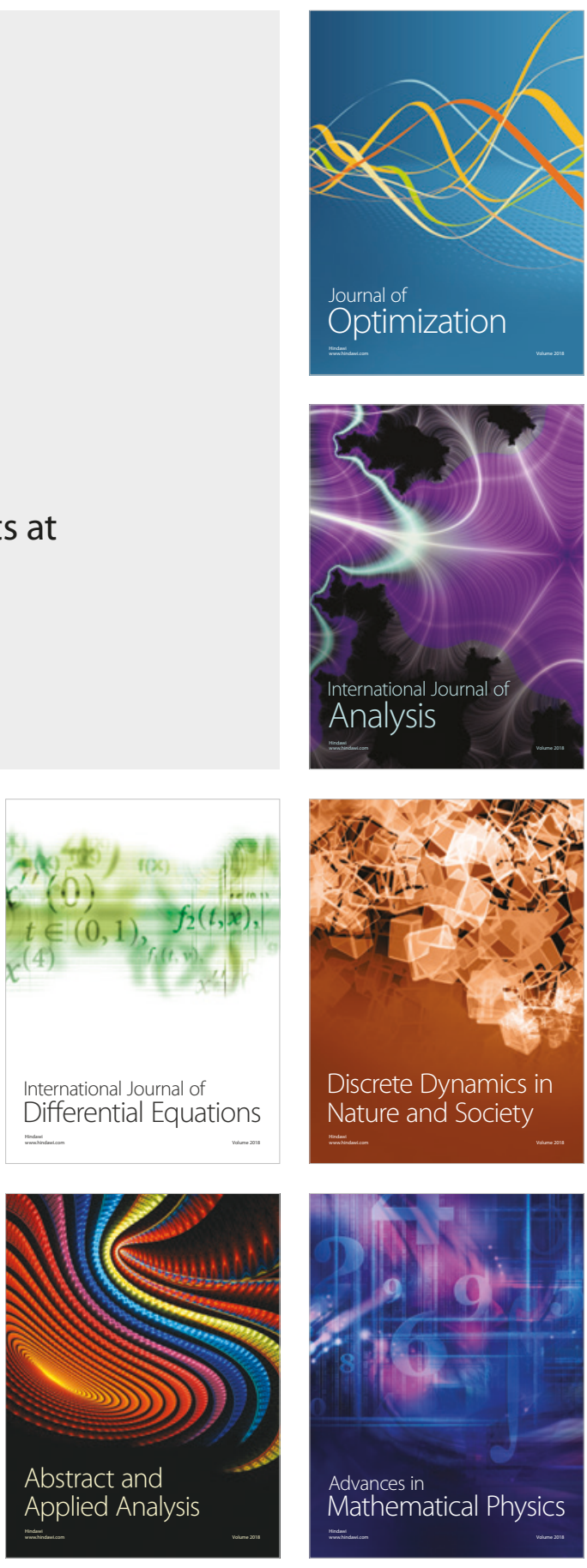